\title{
A unique reason for coronary spasm causing temporary ST elevation myocardial infarction (inferior STEMI) - systemic inflammatory response syndrome after use of pembrolizumab
}

Radomir Nykl ${ }^{1}$, Ondrej Fischer ${ }^{2}$, Karel Vykoupil ${ }^{1}$, Milos Taborsky ${ }^{1}$

\author{
${ }^{1}$ Department of Internal Medicine I - Cardiology, University Hospital, Olomouc, Czech \\ Republic \\ 2Department of Pulmonary Diseases and Tuberculosis, University Hospital, Olomouc, \\ Czech Republic
}

Submitted: 10 October 2017

Accepted: 20 November 2017

Arch Med Sci Atheroscler Dis 2017; 2: e100-e102

DOI: https://doi.org/10.5114/amsad.2017.72531

Copyright (c) 2017 Termedia \& Banach

We present the case of a 71-year-old patient (polymorbid patient with a history of TxN3M1 bronchogenic adenocarcinoma, with no history of cardiovascular disease) who has been indicated for treatment with a new drug - pembrolizumab [1]. He was hospitalized at the Department of Pulmonary Diseases and Tuberculosis (University Hospital, Olomouc, Czech Republic).

This patient started to be subfebrile immediately after the first drug administration, then $3.5 \mathrm{~h}$ later he had chest pain, he felt shortness of breath, objectively he was hypotensive, he had spastic breathing (but with normal saturation), and there was found acute coronary syndrome with persistent ST-segment elevation in the ECG (Figure 1). The patient was habitually treated (acetylsalicylic acid, clopidogrel, heparin), vasopressor support was necessary, and the patient was transported immediately to undergo coronary angiography.

The ECG changes disappeared during the transport to the catheterization laboratory (Figure 2). The patient had no chest pain any longer, he had no problem with breathing then, his saturation was still normal, heart rate around $90 \mathrm{bpm}$ - sinus rhythm, subjectively he only felt weak, objectively there was facial paleness, and spastic respiratory phenomena. He had a normal coronarogram. Ventriculography was also done with normal ejection fraction of the left ventricle (and no signs of tako-tsubo cardiomyopathy). The ECG changes were caused by coronary spasm; the reasons for this are considered below.

He was then admitted to the intensive care unit of the Department of Internal Medicine I - Cardiology. There was a decreasing need for vasopressor support, still normal saturation. Echocardiography was performed - there was normal ejection fraction (both ventricles). Laboratory only minimal positivity of cardioselective markers (maximum value of troponin T 56 ng/l - cut-off 14, maximum value of BNP 1091 pg/ml, cutoff 125). After being stabilized, he was transferred back to the former department to continue the established therapy.

Occurrence of acute heart failure related to pembrolizumab application was already described - but that was caused by myocarditis (premise of cardiotoxicity) [2, 3]. However, in our case, the reason for ECG chang-

\author{
Corresponding author: \\ Radomir Nykl MD \\ Department of Internal \\ Medicine I - Cardiology \\ University Hospital Olomouc \\ 6 I.P.Pavlova St \\ 77900 Olomouc, \\ Czech Republic \\ E-mail: radomir.nykl@gmail. \\ com
}




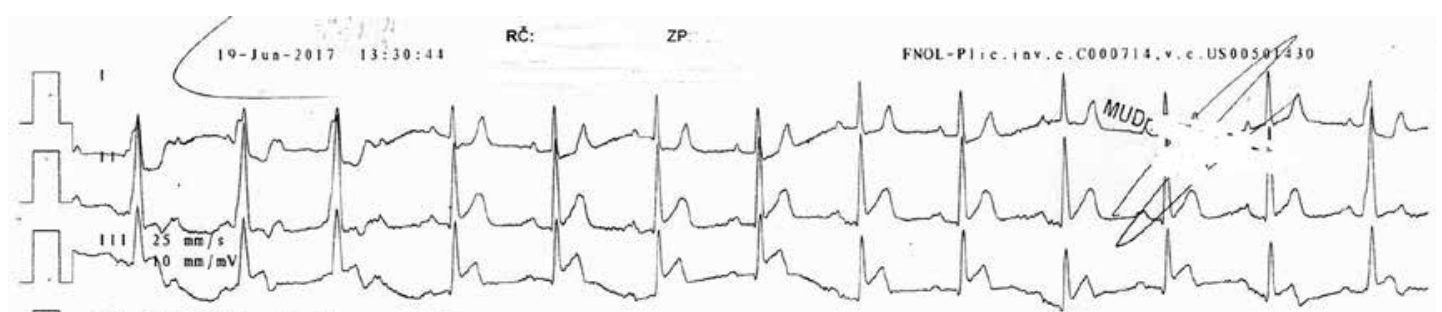

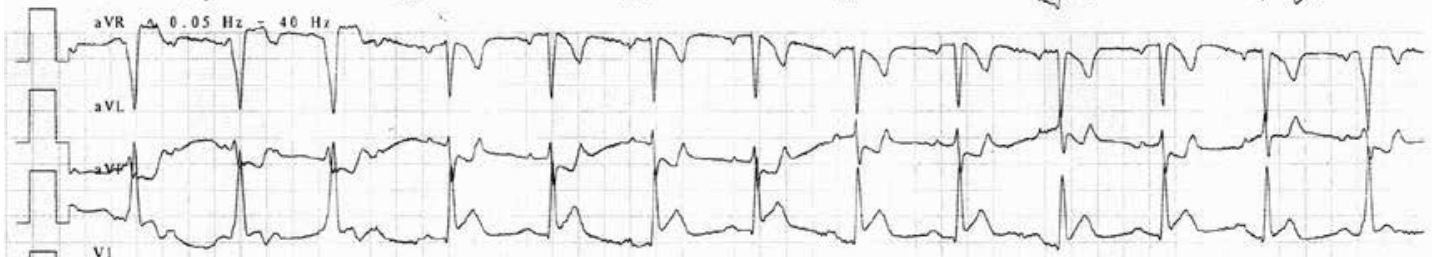

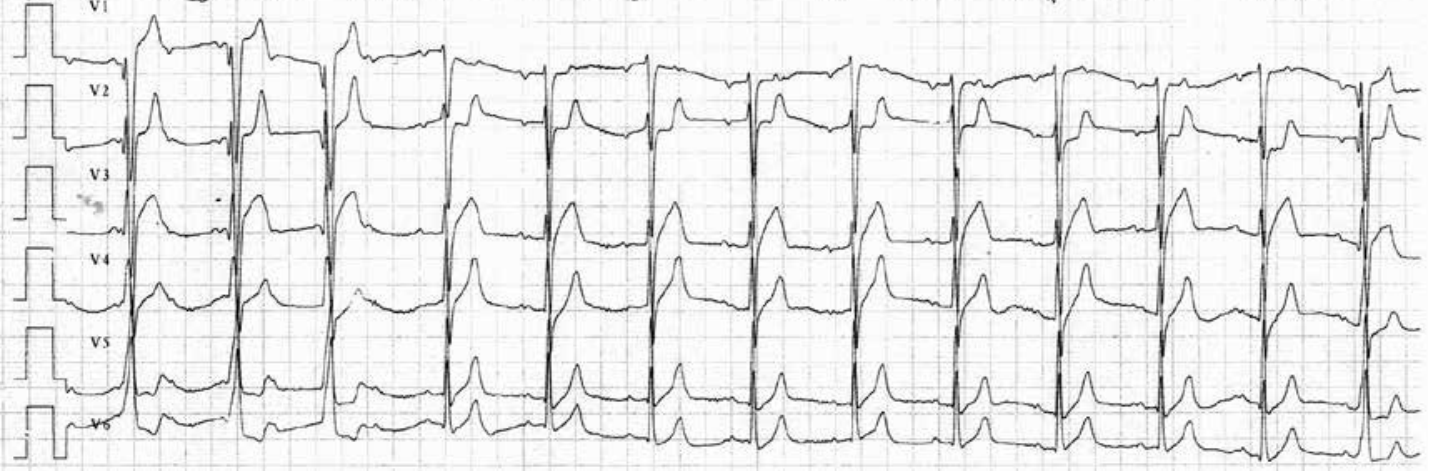

Figure 1. ECG with ST-segment elevations

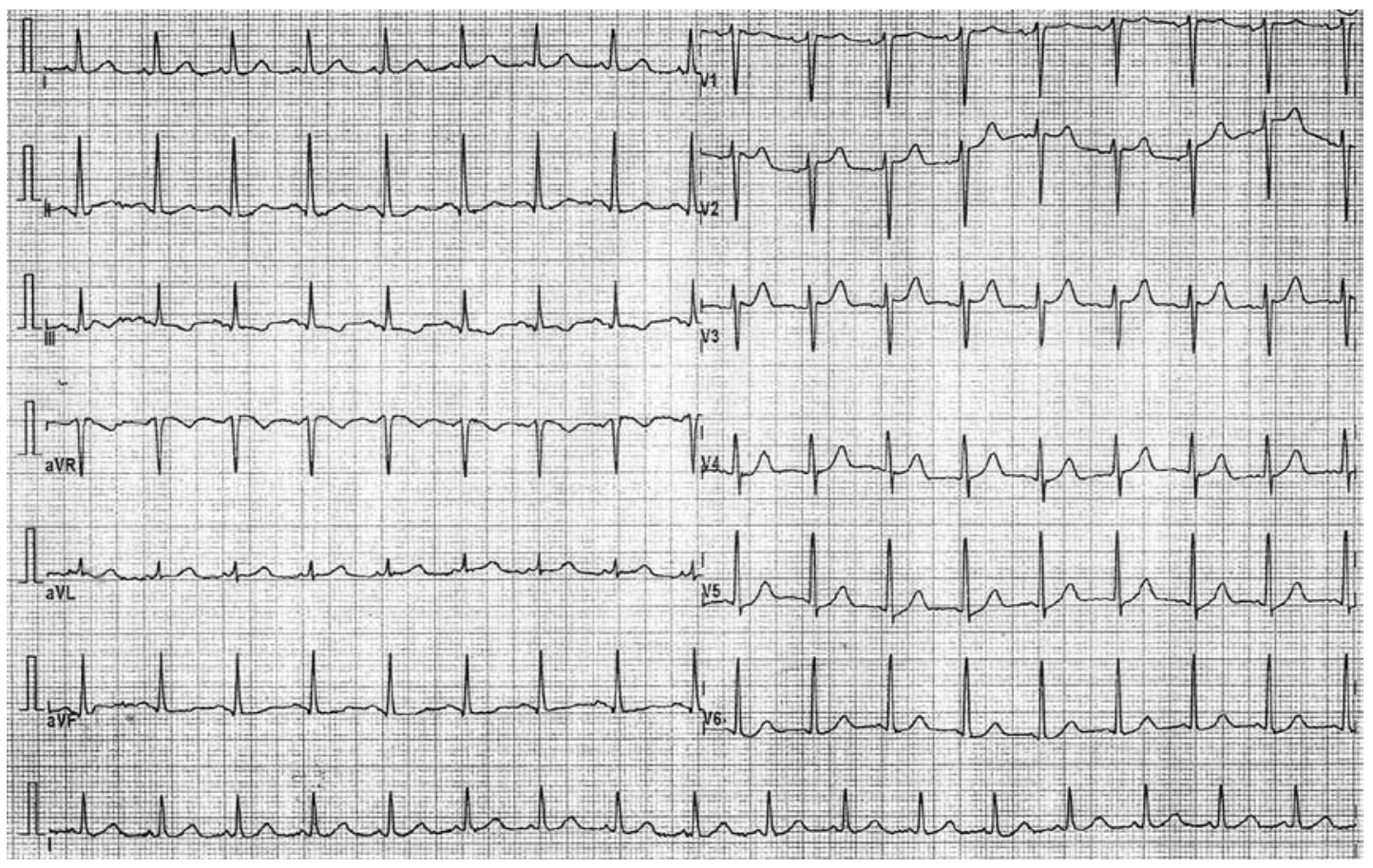

Figure 2. ECG after normalization

es (and the reason for coronary spasm) was the systemic inflammatory response syndrome (SIRS) [4] - use of anti-PDL1 antibody in the predisposed terrain of the activated immune system (premorbid inflammatory infiltration around the tumor ac- cording to the histological findings, premise of the recent infection) as a condition in which the temporary coronary spasm developed. This conclusion is supported by the laboratory findings, the patient's clinical status and the response to the 
therapy (there was rapid improvement of the patient's condition after anti-inflammatory and immunomodulatory therapy). Quick normalization of ECG (within $2 \mathrm{~h}$ ), lack of dynamics of cardiomarkers, normal ventriculography, and echographic and coronarographic findings provide evidence against direct cardiotoxicity of pembrolizumab.

This patient continues with pembrolizumab treatment (always premedicated by non-steroidal antiphlogistics, antihistaminics, antileukotriene therapy, hydration) with no recurrence of any similar problems.

Nowadays, at a time of increasing use of anticancer drugs, we consider it crucial to inform about every potential complication. It is necessary to take all potential risks into account.

\section{Conflict of interest}

The authors declare no conflict of interest.

\section{References}

1. Collin M. Immune checkpoint inhibitors: the battle of giants. Pharm Pat Anal 2017; 6: 135-7.

2. Läubli H, Balmelli C, Bossard M, Pfister O, Glatz K, Zippelius $A$. Acute heart failure due to autoimmune myocarditis under pembrolizumab treatment for metastatic melanoma. J Immunother Cancer 2015; 3: 11.

3. Heinzerling L, Ott PA, Hodi FS, et al. Cardiotoxicity associated with CTLA4 and PD1 blocking immunotherapy. J Immunother Cancer 2016; 4: 50.

4. Zimmer L, Goldinger SM, Hofmann L, et al. Neurological, respiratory, musculoskeletal, cardiac and ocular side-effects of anti-PD-1 therapy. Eur J Cancer 2016; 60: 210-25. 